\title{
Human colorectal cancer-specific CCAT1-L IncRNA regulates long-range chromatin interactions at the MYC locus
}

Jian-Feng Xiang ${ }^{1}$, Qing-Fei Yin ${ }^{1}$, Tian Chen ${ }^{1}$, Yang Zhang ${ }^{1}$, Xiao-Ou Zhang ${ }^{2}$, Zheng Wu ${ }^{1}$, Shaofeng Zhang ${ }^{1}$, Hai-Bin Wang ${ }^{3}$, Junhui Ge ${ }^{3}$, Xuhua Lu ${ }^{3}$, Li Yang ${ }^{2}$, Ling-Ling Chen ${ }^{1}$

${ }^{I}$ State Key Laboratory of Molecular Biology, Institute of Biochemistry and Cell Biology, ${ }^{2}$ Key Laboratory of Computational Biology, CAS-MPG Partner Institute for Computational Biology, Shanghai Institutes for Biological Sciences, Chinese Academy of Sciences, 320 Yueyang Road, Shanghai 200031, China; ${ }^{3}$ Changzheng Hospital, Second Military Medical University, 415 Fengyang Road, Shanghai 200003, China

Cell Research (2014) 24:1150. doi:10.1038/cr.2014.117; published online 1 September 2014

Correction to: Cell Research (2014) 24:513-531. doi:10.1038/cr.2014.35; published online 25 March 2014.

The authors apologized for an error in Supplementary information, Table S2. The ASO sequence used to knockdown CCAT1-L in the study should read as follows:

mCmCmAmCmGTGCACATATTmUmGmAmAmU 